\title{
A Review on the Evolution of Eigenvalue Based Spectrum Sensing Algorithms for Cognitive Radio
}

\author{
Kishor P. Patil \\ Sinhgad Academy of Engineering, S.P. Pune University \\ Pune, India \\ Tel: +91-9545754563 E-mail: kppatil.sae@sinhgad.edu \\ Ashwini S. Lande, Mudassar H. Naikwadi \\ Sinhgad Academy of Engineering, S.P. Pune University \\ Pune, India \\ E-mail: ashwinilande2507@gmail.com \\ E-mail: mhnaikwadi.sae@sinhgad.edu
}

Received: April 21, 2016

DOI: 10.5296/npa.v8i2.9349
Accepted: June 19, 2016

Published: June 30, 2016

URL: http://dx.doi.org/10.5296/npa.v8i2.9349

\begin{abstract}
Spectrum scarcity has been encountered as a leading problem when launching new wireless services. To overcome this problem, cognitive radio is an optimistic solution. Spectrum sensing is a prominent task of cognitive radio. Over the past decade, numerous spectrum sensing algorithms have been proposed. In this paper, we present a comprehensive survey of evolutionary achievements of eigenvalue based spectrum sensing algorithms. The correlation between signal samples due to oversampling, multipath or multiple receivers gets reflected on the eigenvalues of the covariance matrix. It has been observed that different combinations of eigenvalues are used as test statistics and the distribution of eigenvalues and derivation of probability of detection is based on RMT (Random Matrix Theory). The main advantage offered by these algorithms is their robustness to noise uncertainty which severely affect
\end{abstract}


other methods. Furthermore, they do not require accurate synchronization. These detections can be used for different signal detection applications without any prior information of signal or noise. To evaluate the performance of eigenvalue based spectrum sensing techniques under fading channels, we have simulated maximum to minimum eigenvalue based Detection (MME) and maximum eigenvalue based detection (MED) for Rician fading channel. Simulation results shows that MME is much better than MED.

Keywords: Cognitive Radio, Maximum to Minimum Eigenvalue Based Detection, Maximum Eigenvalue Based Detection, Probability of detection, Random Matrix Theory, Spectrum Sensing.

\section{Introduction}

The increasing demand of more and more spectrum for wireless communication has led to extreme spectrum scarcity. The growing spectrum scarcity problem is not because of spectrum band insufficiency; rather it is because of the inefficient utilization of the available spectrum bands [1]. In static spectrum allocation, all of the radio spectrums are allocated to specific services and applications. However, it has been observed that these spectrum bands are mostly underutilized. To improve the spectrum utilization, Cognitive radio is an emerging solution. Cognitive radio is a agile adaptable transceiver which intelligently changes its communication parameters according to the user demands for efficient utilization of radio spectrum [2, 3]. This innovative concept makes the use of vacant spectrum bands possible. Spectrum sensing is one of the most important tasks to evaluate by cognitive radio. It detects the white spaces in spectrum bands and shares it with other unlicensed or secondary users. Multipath fading, time dispersion, shadowing, noise or interference level complicates the spectrum sensing. Spectrum sensing is more difficult in those frequency bands where licensed or primary user can adapt distinct transmission standards, for e.g. ISM band. Reliable and efficient spectrum sensing techniques are needed in order to detect the presence of primary users and vacant spaces. This is because the secondary users need to adjust their operating parameters in order to exploit the unused spectrum. Spectrum sensing techniques can be classified into two main categories non-blind sensing and blind sensing techniques. Priori information of licensed user is necessary for the non-blind techniques and is not necessary for blind spectrum sensing techniques. Energy detection (ED) is a spectrum sensing technique where the received signal energy is calculated and compared with the threshold [4]. Threshold function calculation is based on noise power. Therefore incorrect calculation of noise energy leads to false alarm. Concerning the required knowledge of the noise energy, ED is semi-blind detection technique. Cyclostationary detection (CSD) requires information of cyclic frequencies of received signal detection [5]. The advantage of this technique is that it can detect the signals at very low SNR. The matched filter detection estimation is based on knowledge of received signal signaling feature [6]. Concerning the required knowledge of the signal characteristics, Cyclostationary detection and matched filter are non-blind techniques [7].

Contribution: Most of the detection techniques in the literature require prior knowledge of primary user signal. These techniques are explored in [7]. To overcome the shortcomings 
of non-blind technique different eigenvalue based spectrum sensing techniques have been proposed in the literature. These detection techniques require only the covariance matrix of the received signal rather than signal or noise information. Eigenvalues are calculated from the received covariance matrix. Various methods are available to calculate eigenvalues namely power method [8], inverse power method [8], fast cholesky method [8] and fast iterative methods [9]. In this paper, we give a survey of different eigenvalue based spectrum sensing techniques and also analyze MME and MED performance under Rician fading channel which is not estimated in [10].

Different eigenvalue based detection methods are MME [10], energy with minimum eigenvalue (EME) [10], maximum minus minimum eigenvalue (MMME) [11], difference of means of eigenvalue (DME) [11], LE i.e. MED [12], minimum eigenvalue (ME) [13], two stage spectrum sensing detection (ED and CMME) [14], energy and eigenvalue based detections combined as fully blind and self adaptive [15]. The implementation of this dual stage detection scheme has been carried out using GNU radio and USRP. Here the performance has been analyzed under real noise and interference conditions [16].

The rest of the paper is structured as follows. Section II gives mathematical analysis for distinct eigenvalue based detections. Section III explains about methodologies for various eigenvalue based detections. Section IV shows comparative and diagrammatic representation of distinct eigenvalue based spectrum sensing techniques and evolution of it. Section $\mathrm{V}$ explains Rician fading channel for MME and MED. Section VI explains the results analysis of various detections and simulation result evaluation for MME and MED. Section VII concludes the work and presents some future research improvement.

\section{Mathematical Analysis for Eigenvalue Based Detections}

Binary hypothesis is used for signal detection. Binary hypothesis testing is a method in which we claim the presence or absence of signal. There are two hypothesis namely $H_{0}$ or null hypothesis and $H_{1}$ or one hypothesis. $H_{0}$ is the representation for signal that are not present and $H_{1}$ is the representation for signal and noise both are present. Mathematically this hypothesis can be represented as,

$$
\begin{gathered}
H_{0}=x(n) \\
H_{1}=x(n)+\eta(n)
\end{gathered}
$$

where, $\mathrm{x}(\mathrm{n})$ is signal and $\eta(n)$ is noise. Two probabilities i.e. probability of detection $\left(P_{d}\right)$ and probability of false alarm $\left(P_{f a}\right)$ are of interest for detection. The probability of false alarm defines at the null hypothesis and probability detection at the hypothesis one. Probability of detection also can be defined as $1-P_{f a}$. 


\subsection{System Model}

In order to understand the approach used in adopting RMT for Signal detection, the following system model is used. Considered we are interested in continuous time signal can be given as,

$$
x(t)=s(t)+\eta(t)
$$

where, $s(t)$ is continuous time signal, $\eta(t)$ is noise.

Continuous time signal can be sampled with sampling frequency $F_{s}$ and sampling period is $T_{s}=1 / F_{\text {s. }}$ The Sampled signal can be given as,

$$
x(n T s)=s(n T s)+\eta(n T s)
$$

The notations $x(n T s)=x(n), \quad s(n T s)=s(n), \quad \eta(n T s)=\eta(n)$ are used for simplicity.

The received signal for MIMO system can be given as,

$$
x(n)=\sum_{j=1}^{P} \sum_{k=0}^{N_{i j}} h_{j}(k) s_{j}(n-k)+\eta(n)
$$

where, $P$ is the number of source signals i.e. number of transmitters, $h_{j}(k)$ is channel response and $N_{i j}$ is the order of the channel.

Corresponding notations expressed as in (6), (7) and (8)

$$
\begin{gathered}
x(n)=\left[x_{1}(n), x_{2}(n), \ldots \ldots \ldots ., x_{M}(n)\right]^{T} \\
\eta(n)=\left[\eta_{1}(n), \eta_{2}(n), \ldots \ldots \ldots ., \eta_{M}(n)\right]^{T} \\
h_{j}(n)=\left[h_{1 j}(n), h_{2 j}(n), \ldots \ldots \ldots ., \eta_{M j}(n)\right]^{T}
\end{gathered}
$$

where, $M$ is the oversampling factor. Considering smoothing factor i.e. consecutive windows of length $L$, we can then have an estimation of the sample covariance matrix of the received signal $x(n)$ as ,

$$
R_{x}\left(N_{s}\right)=\frac{1}{N_{s}} \sum_{n=L-1}^{L-2+N s} x(n) * x^{\dagger}(n)
$$

where, $N_{s}$ is number of samples and $x(n)=s(n) * H_{j}+\eta(n)$. It can be further expressed as, 


\section{Macrothink}

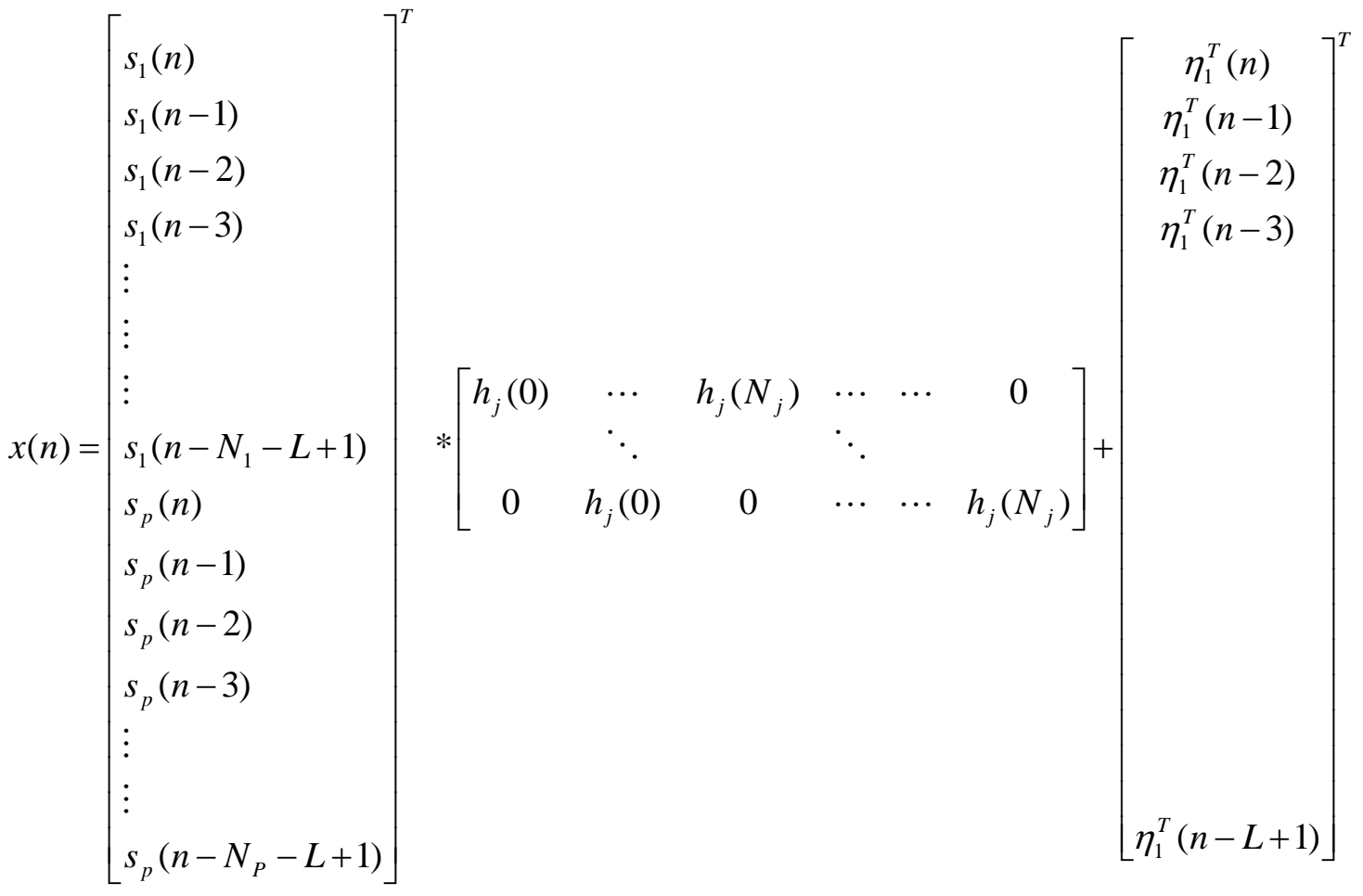

where, $N=\sum_{j=1}^{P} N_{j}$. The matrix dimensions mostly used to calculation given as,

$$
x(n)_{(M L, 1)}=H_{j_{\left(M L,\left(N_{j}+P L\right)\right)}} * s(n)_{(N+P L, 1)}+\eta(n)_{(M L, 1)}
$$

For e.g. If $M=4, L=8, N=9, P=2$ then matrix dimensions will be,

$$
x(n)_{(32,1)}=H_{j_{(32,34)}} * s(n)_{(34,1)}+\eta(n)_{(32,1)}
$$

\subsection{Eigenvalue Estimation Techniques}

Maximum and minimum eigenvalues can be calculated from the received signal covariance matrix. Its complexity increases because of the decomposition of the covariance matrix and the computation of eigenvalues. The computation of eigenvalues is a still open field for research work. Numbers of techniques are available to calculate eigenvalues namely power method [8], inverse power method [8], fast cholesky method [8] and fast iterative methods [9]. Comparative analysis of these methods is shown in Table 1. The impact of $M$, $L$ and $N_{s}$ on the computational complexity is explored in [8,9]. 
Table1. Estimation of computational complexities to calculate eigenvalues

\begin{tabular}{|c|c|}
\hline $\begin{array}{c}\text { Techniques to Estimation } \\
\text { Eigenvalues }\end{array}$ & Computational complexity \\
\hline $\begin{array}{l}\text { Traditional Methods } \\
\text { (MME and EME) }\end{array}$ & $\begin{array}{l}\text { Computational complexity of the sample covariance matrix } \\
=\left(M^{2} L^{2} N_{s}\right) \text { multiplications }+\left(M^{2} L^{2}\left(N_{s}-1\right)\right) \text { addition. If covariance matrix } \\
\text { is block Toeplitz and Hermitian matrix, then computation complexity } \\
=\left(M L N_{s}\right) \text { multiplications }+\left(M L\left(M N_{s}-1\right)\right) \text { additions. Complexity for } \\
\text { calculation of the eigenvalues }=O\left(M^{3} L^{3}\right) \text { (multiplications + additions). } \\
\text { Then the total computational complexity of traditional method = } \\
\left(M L N_{s}\right)+O\left(M^{3} L^{3}\right) \text {. }\end{array}$ \\
\hline Power Method & $\begin{array}{l}\text { It approximates the largest eigenvalue of a symmetric positive definite } \\
\text { matrix. } \\
\text { Computational complexity of power method }=O(k M L) \\
\text { where, } k \text { is the number of operations under a particular error threshold. }\end{array}$ \\
\hline Inverse Power Method & $\begin{array}{l}\text { It approximates the smallest eigenvalue without sorting and finding all } \\
\text { eigenvalues. } \\
\text { Computational complexity of inverse power method }=O\left(M^{4} L^{4}\right) \text { using } \\
\text { naive gaussian elimination. If covariance matrix is Hermitian, then } \\
\text { computational complexity }=O\left(M^{3} L^{3} / 3\right) \text {. Therefore the total complexity } \\
=O\left(M^{3} L^{3}\right) \text {. }\end{array}$ \\
\hline Fast Cholesky Method & $\begin{array}{l}\text { Various well-known algorithms like Levinson and Schur algorithm } \\
\text { which factorize Toeplitz with computational complexity less than } \\
O\left(M^{2} L^{2}\right) \text {. The Schur algorithm exploits parallel computing to reduce its } \\
\text { computational latency and detail algorithm explained in [8] finding } \\
\text { Cholesky factor of Toeplitz matrices. }\end{array}$ \\
\hline Fast Iterative Methods & 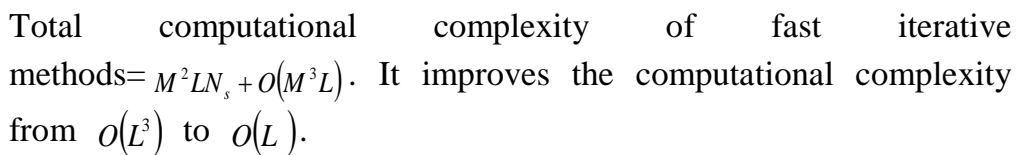 \\
\hline
\end{tabular}

\section{Methodologies for Eigenvalue Based Detections}

\subsection{Maximum-Minimum Eigenvalue detection}

MME and EME are used to perform signal detection without prior knowledge of signal or noise power. MME detection compares the threshold with the ratio of the maximum eigenvalue to the minimum eigenvalue. EME detection compares the threshold with the value of the ratio of the average signal power to the minimum eigenvalue. Unlike ED, here the threshold is not based on noise power. In these algorithms, the threshold is estimated by using number of samples, smoothing factor and false alarm probability [10].

Step 1. Compute the sample covariance matrix of the received signal. 


$$
R_{x}\left(N_{s}\right)=\frac{1}{N_{s}} \sum_{n=L-1}^{L-2+N s} x(n) * x^{\dagger}(n)
$$

Step 2. Calculate the maximum and minimum eigenvalue of the sample covariance matrix i.e. $\lambda_{\max }$ and $\lambda_{\min }$.

Step 3. Computation of the threshold can be given as,

$$
\gamma=\frac{\left(\sqrt{N_{s}}+\sqrt{M L}\right)^{2}}{\left(\sqrt{N_{s}}-\sqrt{M L}\right)^{2}} *\left(1+\frac{\left(\sqrt{N_{s}}+\sqrt{M L}\right)}{\left(N_{s} M L\right)} F^{-1}\left(1-P_{f a}\right)\right)
$$

where, $F^{-1}$ is Tracy-Wisdom distribution.

Step 4. Compare the ratio of maximum eigenvalue to minimum eigenvalue with the threshold, if $\lambda_{\max } / \lambda_{\min }>\gamma$ then licensed user exists otherwise channel is vacant for unlicensed users.

Tracy-Widom Distribution: To study the eigenvalue distributions of a random matrix the joint probability density function (PDF) of ordered eigenvalues of a Wishart random matrix is used. The expression of the PDF is very complicated. To address this problem, the Tracy-Widom distribution is used. The distribution function is defined as,

$$
F_{1}(t)=\exp \left(-\frac{1}{2} \int_{t}^{\infty}\left(q(u)-(u-1) q^{2}(u) d u\right)\right)
$$

where, $\mathrm{q}(\mathrm{u})$ is the solution of the nonlinear Painleve II differential equation,

$$
q^{\prime \prime}=u q(u)+2 q^{3}(u)
$$

It is difficult to evaluate. Therefore tables or MATLAB codes are used to compute it. Table 2 gives the values of $F_{1}$ at some points.

Table 2: Numerical Table For the Tracy-Widom Distribution of Order 1

\begin{tabular}{|l|l|l|l|l|l|l|l|l|l|}
\hline $\mathrm{t}$ & -3.90 & -3.18 & -2.78 & -1.91 & -1.27 & -0.59 & 0.45 & 0.98 & 2.02 \\
\hline$F_{1}(\mathrm{t})$ & 0.01 & 0.05 & 0.10 & 0.30 & 0.50 & 0.70 & 0.90 & 0.95 & 0.99 \\
\hline
\end{tabular}

Step 5. The probability of detection of the MME detection is,

$$
P_{d}=1-F_{1}\left(\frac{\gamma^{*} N_{s}+N_{s}\left(\gamma \rho_{M L}-\rho_{1}\right) / \sigma_{n}^{2}-\mu}{v}\right)
$$


where, $\rho_{M L}$ and $\rho_{1}$ are minimum and maximum eigenvalues of signal and channel respectively i.e. $\left(H R_{s} H^{\dagger}\right), \sigma_{n}^{2}$ is noise variance.

Step 6. The probability of false alarm of the MME detection is,

$$
P_{f a}=1-F_{1}\left(\frac{\gamma\left(\sqrt{N_{s}}-\sqrt{M L}\right)^{2}-\mu}{v}\right)
$$

where, $\mu=\left(\sqrt{N_{s}-1}+\sqrt{M L}\right)^{2}, \quad v=\left(\sqrt{N_{s}-1}+\sqrt{M L}\right) *\left(\frac{1}{N_{s}-1}+\frac{1}{\sqrt{M L}}\right)^{1 / 3}$.

\subsection{Energy with Minimum Eigenvalue Detection}

Step1. The same as given in MME detection.

$$
T\left(N_{s}\right)=\sum_{i=1}^{M} \sum_{n=0}^{N_{s}-1}\left|x_{i}(n)\right|^{2}
$$

Step2. Calculate the average power of the received signal $T\left(N_{s}\right)$ and the minimum eigenvalue $\lambda_{\text {min }}$ of the received covariance matrix.

Step 3. Computation of threshold can be given as,

$$
\gamma=\left(\sqrt{\frac{2}{M N_{s}}} Q^{-1}\left(P_{f a}\right)+1\right) *\left(\frac{N_{s}}{\left(\sqrt{N_{s}}-\sqrt{M L}\right)^{2}}\right)
$$

where, $\mathrm{Q}($.$) is the inverse \mathrm{Q}$ function.

Step 4. Compare the ratio of average power to the minimum eigenvalue with the threshold, if $T\left(N_{s}\right) / \lambda_{\min }>\gamma$ then licensed user exists otherwise licensed user does not exist.

Step 5. For the EME method, the probability of detection is,

$$
P_{d}=Q\left(\frac{\gamma\left(\rho_{M L}+\frac{\sigma_{n}^{2}}{\sqrt{N_{s}}}\left(\sqrt{N_{s}}-\sqrt{M L}\right)\right)-\left(\frac{T_{r}\left(H R_{s} H^{\dagger}\right)}{M L}\right)-\sigma_{n}^{2}}{\sqrt{\frac{2}{M N_{s}}} * \sigma_{n}^{2}}\right)
$$

where, $Q($.$) is the \mathrm{Q}$ function, $T_{r}($.$) is the trace of a matrix.$ 


\section{Macrothink}

Step 6. For the EME method, the probability of false alarm is,

$$
P_{f a}=Q\left(\frac{\gamma \sqrt{M}\left(\sqrt{N_{s}}-\sqrt{M L}\right)^{2}-N_{s} \sqrt{M}}{\sqrt{2 N_{s}}}\right)
$$

\subsection{Maximum Minus Minimum Eigenvalue Detection}

For higher noise, MME performance gets affected by SNR. To tackle this issue, MME can be modified as MMME. In MMME detection, maximum minus minimum eigenvalue is compared with the threshold for test static [11].

Step1. The same as given in MME Detection.

Step 2. Calculate the maximum and minimum eigenvalue from the covariance matrix i.e.

$\lambda_{\max }$ and $\lambda_{\min }$ and compute $T_{M M M E}$,

$$
T_{M M M E}=\ln \left(\frac{\lambda_{\max }}{\lambda_{\min }}\right)=\lambda_{\text {max }}-\lambda_{\text {min }}
$$

Step 3. Compare $T_{M M M E}$ with the threshold, if $T_{M M M E}>\gamma$ then licensed user exists else channel is available for unlicensed users.

\subsection{Difference of Means of Eigenvalue Detection}

A typical phenomenon to decrease noise is to average over multiple values. MMME does not exploit this phenomenon. Therefore, MMME can be modified as DME detection. In DME detection, difference of means of eigenvalue is compared with the threshold for test static [11].

Step1. Compute TDME using given equation,

$$
T_{D M E}\left(N_{1}\right)=\frac{1}{N_{s}} \sum_{i=1}^{N_{1}} \hat{\lambda}-\left(\frac{1}{M L-N_{1}}\right) \sum_{i=N_{1}+1}^{M L} \hat{\lambda}_{i}
$$

where, $\hat{\lambda}$ are eigenvalues. The mean of $T_{D M E, H_{0}}$ and $T_{D M E, H_{1}}$ can be minimized and maximized respectively by setting the value of $N_{1}$. Therefore it is necessary to set the value of $N_{1}$ in an analytic way as it has a great impact on DME performance. 
Step 2. Calculate the threshold $\gamma$ and Compare $T_{D M E}$ with the threshold, if $T_{D M E}>\gamma$ then channel is occupied else channel is vacant and available for unlicensed users.

\subsection{Maximum Eigenvalue Based Detection for Correlated Noise}

Most of the eigenvalue based techniques assume the presence of white noise at the receiver. However, in practice, noise can be correlated because of imperfections in filtering interference or oversampling operations. Therefore maximum or large eigenvalue based spectrum sensing is evaluated for correlated noise. LE detection compares the ratio of maximum eigenvalue to noise power with the threshold. It is observed that the threshold calculated for uncorrelated scenario when applied to correlated scenarios, the $P_{f a}$ value gets deviated from the targeted $P_{f a}$ value. To address this problem, threshold function is derived for correlated noise [12].

Step1. The same as given in MME detection.

Step2. Calculate the maximum eigenvalue of the covariance matrix i.e. $\lambda_{\max }$ and noise power $\sigma_{n}^{2}$

Step 3. Compute the threshold for correlated noise.

$$
\gamma=\frac{\sigma_{c}^{2} F_{2}^{-1}\left(1-P_{f a}\right)+N_{s}^{2 / 3} * v_{c}}{N_{s}^{2 / 3}}
$$

where, $F_{2}^{-1}$ is Tracy-Widom distribution of order $2, \sigma_{c}^{2}$ and $v_{c}$ can be calculate as given in [12].

Step 4. Compare the ratio of maximum eigenvalue to noise power with the threshold, if $\lambda_{\max } / \sigma_{n}^{2}>\gamma$ then signal is present otherwise signal is absent.

Step 5. The probability of detection of the LE detection is,

$$
P_{d}=F_{2}\left(N_{s}^{2}{ }^{2 / 3}\left(\frac{\gamma-v_{c}}{\sigma_{c}^{2}}\right)\right)
$$

Step 6. The probability of false alarm of the LE detection is,

$$
P_{f a}=1-F_{2}\left(N_{s}^{2 / 3}\left(\frac{\gamma-v_{c}}{\sigma_{c}^{2}}\right)\right)
$$




\subsection{Minimum Eigenvalue Detection}

Practically, at a particular SNR level the fluctuation of maximum eigenvalues is always more than minimum eigenvalues. This results in more minimum eigenvalues falling above its threshold. This in turn leads to increased probability of detection than maximum eigenvalue based detection. In ME detection, the ratio of the minimum eigenvalue to noise power is used as the test statistic [13].

Step 1. The same as given in MME detection.

Step 2. Calculate the minimum eigenvalue of the covariance matrix i.e. $\lambda_{\min }$.

Step 3. Compute the threshold $\gamma$ and noise power $\sigma_{n}^{2}$. (Theoretically threshold is 1 ).

Step 4. Compare the ratio of minimum eigenvalue to noise power with the threshold, if $\lambda_{\min } / \sigma_{n}^{2}>\gamma$ then signal is present otherwise signal is absent.

\subsection{Two Stage Spectrum Sensing Detection}

A combination of two spectrum sensing techniques which explores the advantages of each individual detector can be used for increasing sensing efficiency. ED performance is better at high SNR, whereas CSD and eigenvalue based detection perform better at low SNRs. Therefore in dual stage detection, ED is used as first stage and eigenvalue or CSD is used as second stage. There is a novel two stage spectrum sensing detection where ED is used as coarse sensing and CMME (Combination of Maximum and Minimum Eigenvalue) is used as fine sensing. Here a dual stage threshold parameter is designed for the maximization of detection probability [14].

Step 1. Compute the energy of received signal.

$$
D^{c}=\sum_{n=1}^{M^{c}} x(n)^{2}
$$

where, $M^{c}$ is the number of samples in ED.

Note: $(.)^{c}$ shows first stage detection and $(.)^{f}$ shows second stage detection.

Step 2. Threshold for ED detection can be given as,

$$
\gamma=f\left(\gamma_{2}\right)=Q^{-1}\left(\frac{\beta-\left(1-F_{1}\left(\frac{\gamma^{\prime}\left(\sqrt{N_{s}}+\sqrt{K}\right)^{2}-\mu}{v}\right)\right)}{1-\left(1-F_{1}\left(\frac{\gamma^{\prime}\left(\sqrt{N_{s}}-\sqrt{K}\right)^{2}-\mu}{v}\right)\right)}\right) *\left(\sqrt{2 M^{c} \sigma_{n}^{4}}\right)+\left(M^{c} \sigma_{n}^{2}\right)
$$


where, $K$ is secondary or unlicensed user and $(K \geq 1), \quad \beta=P_{f a}^{c}\left(1-P_{f a}^{c}\right) * P_{f a}^{f}, \quad \gamma^{\prime}=1 / \gamma_{2}-1$.

Step 3. Compare the energy of received signal with the threshold, if $D^{c}>\gamma$ then licensed user is present else switch to the fine sensing stage.

Step 4. For the ED method, the probability detection is

$$
P_{d}^{c}=Q\left(\frac{\gamma-M^{c}\left(\sigma_{n}^{2}+\sigma_{s}^{2}\right)}{\sqrt{2 M^{c}\left(\sigma_{n}^{2}+\sigma_{s}^{2}\right)^{2}}}\right)
$$

where, $\sigma_{s}^{2}$ is signal variance.

Step 5. For the ED method, the probability of false alarm is

$$
P_{f a}^{c}=Q\left(\frac{\gamma-M^{c} \sigma_{n}^{2}}{\sqrt{2 M^{c} \sigma_{n}^{4}}}\right)
$$

Step 6. Calculate the maximum and minimum eigenvalue of the covariance matrix i.e. $\lambda_{\max }$ and $\lambda_{\min }$.

Step 7. For the MME method, computation of threshold can be given as,

$$
\max _{\gamma_{2}}=P_{d}\left(f\left(\gamma_{2}\right), \gamma_{2}\right)
$$

where, $\gamma_{2}$ and $f\left(\gamma_{2}\right)$ can be calculated as given in [14].

Step 8. $\lambda_{\max } /\left(\lambda_{\max }-\lambda_{\min }\right)$ is compared with the threshold, if $\lambda_{\max } /\left(\lambda_{\max }-\lambda_{\min }\right)>\gamma_{2}$ then licensed user exists otherwise channel is vacant for unlicensed user.

Step 9. For the CMME method, the Probability detection is,

$$
\left.P_{d}^{f}=Q\left(\frac{\gamma_{2}-\left(\frac{\lambda_{\text {max }}}{\lambda_{\text {max }}-\lambda_{\text {min }}}\right)}{\sqrt{4 N_{s}\left(\frac{\lambda_{\max }}{\lambda_{\text {max }}-\lambda_{\min }}\right)}}\right)\right)
$$

Step 10. For the CMME method, the probability of false alarm is,

$$
P_{f a}^{f}=1-F_{1}\left(\frac{\gamma^{\prime}\left(\sqrt{N_{s}}-\sqrt{K}\right)^{2}-\mu}{v}\right)
$$


3.8 Energy and Eigenvalue-Based Combined Fully-Blind Self-Adapted Detection

Dual stage detection of ED and MME can be modified as fully blind and self-adaptive. Noise estimation is carried out at the MME detection and this information is fed back to energy detector to adjust its detection threshold [15].

Step 1.Compute the energy of received signal.

$$
X=\sum_{n=1}^{N_{s}}\left|x_{n}\right|^{2}
$$

Step 2.For the ED method, threshold can be given as,

$$
\rho=\sqrt{2 N_{s}} * \sigma_{z}^{2} Q^{-1}\left(P_{f a}^{c}\right)+N_{s} \sigma_{z}^{2}
$$

where, $\sigma_{z}^{2}=\sigma_{n}^{2}$

Step 3. The energy of received signal is compared with the threshold, if $X>\rho$ then licensed user is present else switch to the fine sensing stage.

Step 4. For the ED method, the probability detection is,

$$
P_{d}^{c}=Q\left(\frac{\rho-N_{s}(S N R+1) \sigma_{z}^{2}}{2 \sqrt{2 N_{s}}(S N R+1) \sigma_{z}^{2}}\right)
$$

Step 5. For the ED method, the probability of false alarm is,

$$
P_{f a}^{c}=Q\left(\frac{\rho-N_{s} \sigma_{z}^{2}}{2 \sqrt{2 N_{s}} \sigma_{z}^{2}}\right)
$$

Step 6.Compute the sample covariance matrix of the received signal.

Step 7.Calculate the maximum and minimum eigenvalue from covariance matrix i.e. $\lambda_{\max }$ and $\lambda_{\min } \cdot$

Step 8. For the MME method, computation of threshold can be given as,

$$
\gamma=\left(\frac{\sqrt{N_{s}}-\sqrt{L}}{\sqrt{N_{s}}-\sqrt{L}}\right)^{2}\left(1+\left(\frac{\left(\sqrt{N_{s}}+\sqrt{L}\right)^{-2 / 3}}{\left(N_{s} L\right)^{1 / 6}}\right) F^{-1}\left(1-P_{f a}^{f}\right)\right)
$$

Step 9. Compare $\lambda_{\max } / \lambda_{\min }$ with the threshold, if $\lambda_{\max } / \lambda_{\min }>\gamma$ then licensed user exists otherwise channel is vacant for unlicensed user. 
Step 10. Probability of detection is same as MME detection.

Step 11. Probability of false alarm can be calculated by rearranging the threshold function.

Step 12. Estimate Noise at the MME detection and go to step 1.

\section{Comparative Analysis}

\subsection{Comparative Table for Distinct Eigenvalue Based Detection}

Table 3: Comparison of Eigenvalue Based Detection Algorithms

\begin{tabular}{|c|c|c|}
\hline \multirow{2}{*}{$\begin{array}{l}\text { Detection } \\
\text { Methods }\end{array}$} & \multicolumn{2}{|l|}{ Comparison Parameters } \\
\hline & Computational Complexity & Accuracy \\
\hline MME & $\begin{array}{l}\text { ED requires } M N_{s} \text { multiplications }+M\left(N_{s}-1\right) \text { additions. MME } \\
\text { requires } M^{2} L N_{s} \text { multiplications }+M^{2} L\left(N_{s}-1\right) \text { additions for } \\
\text { calculation of covariance matrix and } O(M L)^{3} \text { (multiplications } \\
\text { +additions) for the calculation of eigenvalues. Computational } \\
\text { complexity of MME is } M L \text { times more than ED. }\end{array}$ & $\begin{array}{l}\text { More accurate than ED even if } \\
\text { it does not require any prior } \\
\text { information about signal and } \\
\text { noise power. }\end{array}$ \\
\hline EME & Computation complexity of EME is same as MME. & $\begin{array}{l}\text { Highly accurate only when } \\
\text { average power is calculated } \\
\text { correctly. }\end{array}$ \\
\hline MMME & Computation complexity of MMME is almost same as MME. & $\begin{array}{l}\text { Accuracy is more than MME at } \\
\text { high SNR also. }\end{array}$ \\
\hline DME & $\begin{array}{l}N 1 \text { has a huge effect on the performance of the DME detector. } \\
\text { Therefore the specific choice of } N 1 \text { increases computational } \\
\text { complexity of DME. }\end{array}$ & $\begin{array}{l}\text { Accuracy is more than MME } \\
\text { and MMME. }\end{array}$ \\
\hline LE & $\begin{array}{l}\text { Computational complexity of LE is more than MME, EME, } \\
\text { MMME and DME as threshold calculation of correlated noise } \\
\text { is depend on correlated noise power and } v_{c} \text {. }\end{array}$ & $\begin{array}{l}\text { It gives better accuracy for } \\
\text { uncorrelated noise than } \\
\text { correlated noise. }\end{array}$ \\
\hline $\mathrm{ME}$ & $\begin{array}{l}\text { ME requires } O\left(L^{2} N_{s}\right) \text { operations for calculating covariance } \\
\text { matrix and } O\left(L^{3}\right) \text { for calculating eigenvalue. Total } \\
\text { complexity }=O\left(L^{2} N_{s}\right)+O\left(L^{3}\right) \text {. }\end{array}$ & $\begin{array}{l}\text { Dependency on the accurate } \\
\text { calculation of noise power } \\
\text { decreases the accuracy of ME } \\
\text { than MME, MMME and DME }\end{array}$ \\
\hline ED+CMME & $\begin{array}{l}\text { ED +CMME includes computational complexity of ED and } \\
\text { CMME which is greater than MME, MMME, DME, EME and } \\
\text { LE. }\end{array}$ & $\begin{array}{l}\text { Accuracy is better than single } \\
\text { stage detection technique as it } \\
\text { exploits advantages of ED and } \\
\text { MME detector. }\end{array}$ \\
\hline $\begin{array}{l}\text { Fully-Blind } \\
\text { and Self } \\
\text { Adaptive } \\
\text { ED+MME }\end{array}$ & $\begin{array}{l}\text { ED requires } N_{s} \text { multiplications and }\left(N_{s}-1\right) \text { additions. Total } \\
\text { complexity of } \mathrm{ED}=O\left(N_{s}\right) \text {. MME requires } N_{s} \text { multiplications } \\
\text { and }\left(N_{s}-1\right) \text { additions for sample covariance matrix } \\
\text { calculation and } O\left(L^{3}\right) \text { operations for finding eigenvalues. } \\
\text { Therefore, total complexity MME }=O\left(N_{s}\right)+O\left(L^{3}\right) \text {. Total } \\
\text { complexity=(ED+MME) complexity }\end{array}$ & $\begin{array}{l}\text { Accuracy is more than } \\
\text { ED+MME detection technique. }\end{array}$ \\
\hline
\end{tabular}




\subsection{Comparative Diagrams}

Different eigenvalue based spectrum sensing algorithms are shown in Fig.1. Different combinations of eigenvalues are used for signal detection. For each of the detectors, a test-statistic $\mathrm{T}$ is given. The test static compares with the threshold. On the basis of this result, detector decides presence or absence of licensed user.

$$
\begin{aligned}
& \text { If } T>\gamma \text { then } H_{1} \\
& \text { If } T<\gamma \text { then } H_{0}
\end{aligned}
$$

if the test statistic $(\mathrm{T})$ is greater than the threshold , then licensed user is present. If it is lesser, then licensed user is not present.

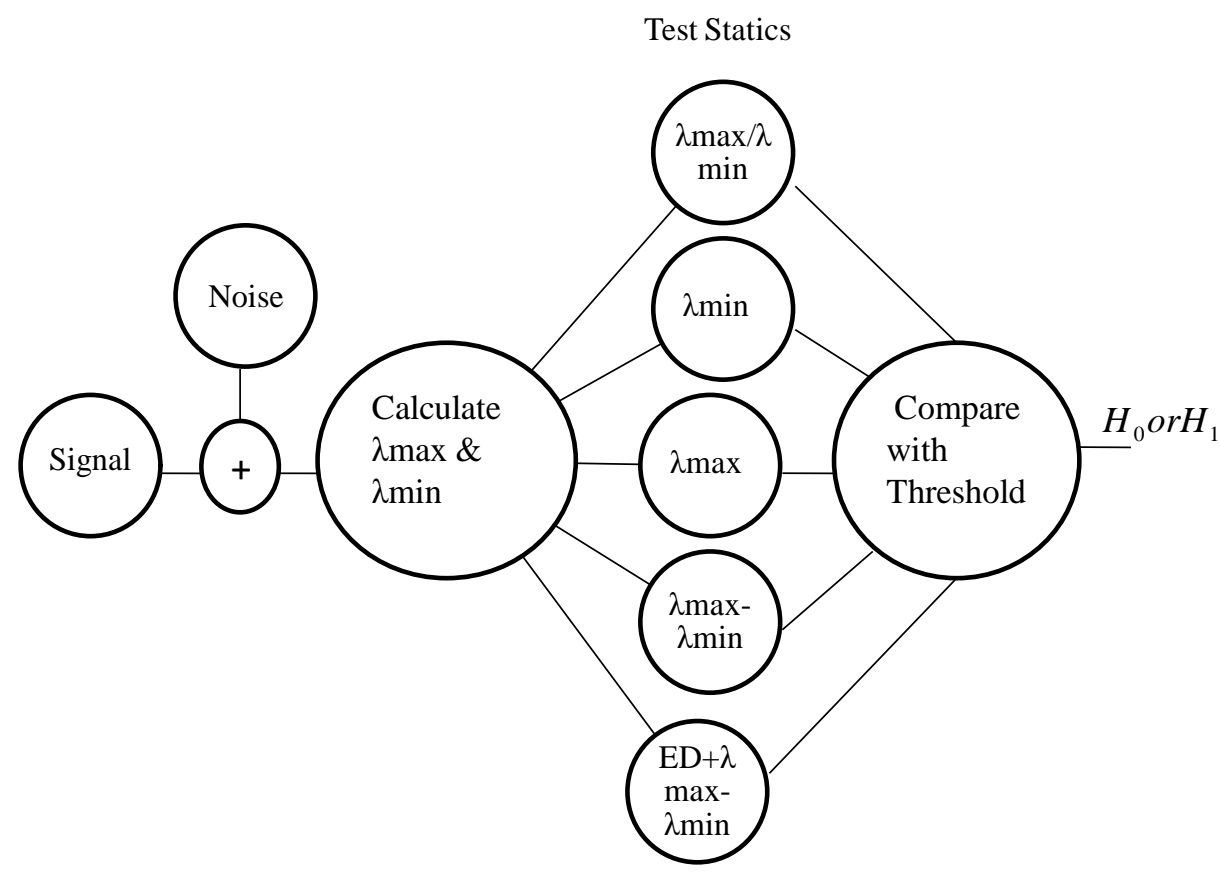

Figure 1: Methodology for Different Eigenvalue Based Spectrum Sensing

Fig. 2 shows the evolution of eigenvalue Based spectrum Sensing Techniques. The first level represents MME and EME detection to perform signal detection fully blind. The second level represents modification of MME as MMME and DME to overcome disadvantage of 


\section{Macrothink}

Network Protocols and Algorithms

ISSN 1943-3581

2016, Vol. 8, No. 2

MME. The third level represents LE detection for correlated noise to get targeted probability of false alarm value. The fourth level represents $\mathrm{ME}$ to avoid fluctuation of maximum eigenvalue over particular value of SNR. The fifth level represents two stage detection to get maximum probability of detection over individual detection.

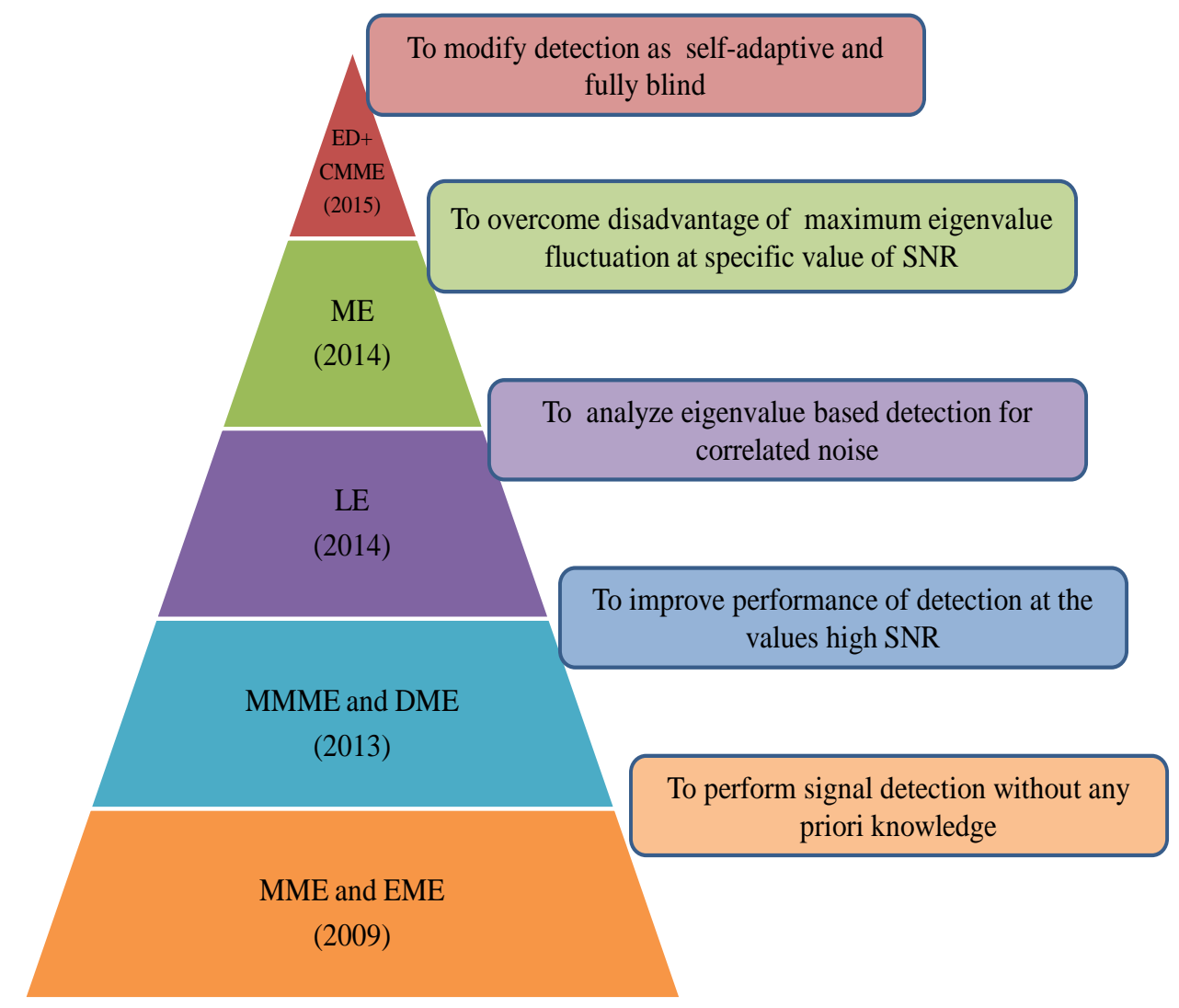

Figure 2: Evolution of Eigenvalue Spectrum Sensing Techniques

\section{Rician Fading Channel for MME and MED}

Due to the reflection, diffraction, scattering etc. the strength of the received signals follow the Rician distribution, we called this channel as Rician fading channel. It can be described at the $j$ th time instant as,

$$
h_{j}=\sqrt{\left(a_{j}+\rho\right)^{2}+\left(b_{j}\right)^{2}}
$$

where, $\rho$ is the amplitude, $a_{j}$ and $b_{j}$ are two Gaussian random variables with variance $\sigma_{0}^{2}$ and mean zero. The PDF (Probability Density Function) of Rician channel can be given as, 


$$
p(h)=\frac{h}{\sigma_{0}^{2}} \exp \left[\frac{-\left(-h^{2}+\rho^{2}\right)}{2 \sigma_{0}^{2}}\right] I_{0}\left[\frac{h \rho}{\sigma_{0}^{2}}\right]
$$

where, $I_{0}($.$) is the zero-order modified Bessel function of the first kind.$

To analyze simulation performance of MED and MME under Rician fading channel in MATLAB, we have generated the uncorrelated fading sequences of the Rician channel distribution. The mean-squared value of the Rician distribution is $2 \sigma_{0}^{2} \sqrt{K+1}$. We generally require the Rician distribution with unit mean-squared value to make the signal power and SNR corresponding. Therefore (37) can rewrite as,

$$
h_{j}=\sqrt{\frac{\left(a_{j}+\sqrt{2 K}\right)+b_{j}}{2(K+1)}}
$$

where, $\mathrm{K}$ is Rician factor. It can be defined as, $K=\rho^{2} / 2 \sigma_{0}^{2}$

\section{Discussion}

In this section, we will discuss the effect of number of samples and smoothing factor on $P_{d}$ and $P_{f a}$ of different eigenvalue based spectrum sensing detections. For ME detection the $P_{d}$ increases with the number of samples more rapidly than MME detection. For the $P_{f a}$, eigenvalue based algorithms do not change much with number of samples. It is seen that both $P_{d}$ and $P_{f a}$ of the MME slightly increase with $L$, but the effect of $L$ on $P_{d}$ of ME detection is comparatively greater than MME. For LE method, the probability of detection improves the sensing performance and achieves perfect sensing performance above SNR value of 10dB. MMME detector has better sensing performance than MME, while the DME detector shows the best performance over MMME and MME. The $P_{d}$ of DME is comparatively less than the $P_{d}$ of two stage detection. The probability of detection performance of two-stage spectrum sensing using ED and CMME is better compared to two-stage spectrum sensing using ED and CSD. ED and CMME gives $0.79 P_{d}$ while ED and CSD gives $0.59 P_{d}$ at $-30 \mathrm{~dB}$.

\subsection{Simulation Result Analysis}

In the simulation analysis, we use the randomly generated signal as the primary signal through Rician fading channel, and then simulate it in MATLAB. We compare and analyze the simulation results of MME and MED. We set the matrix length as $L=8$, use the number of samples $N_{s}=100000$, vary SNR from -20 to $0 \mathrm{~dB}$ and simulate 100 Monte Carlo realizations. 


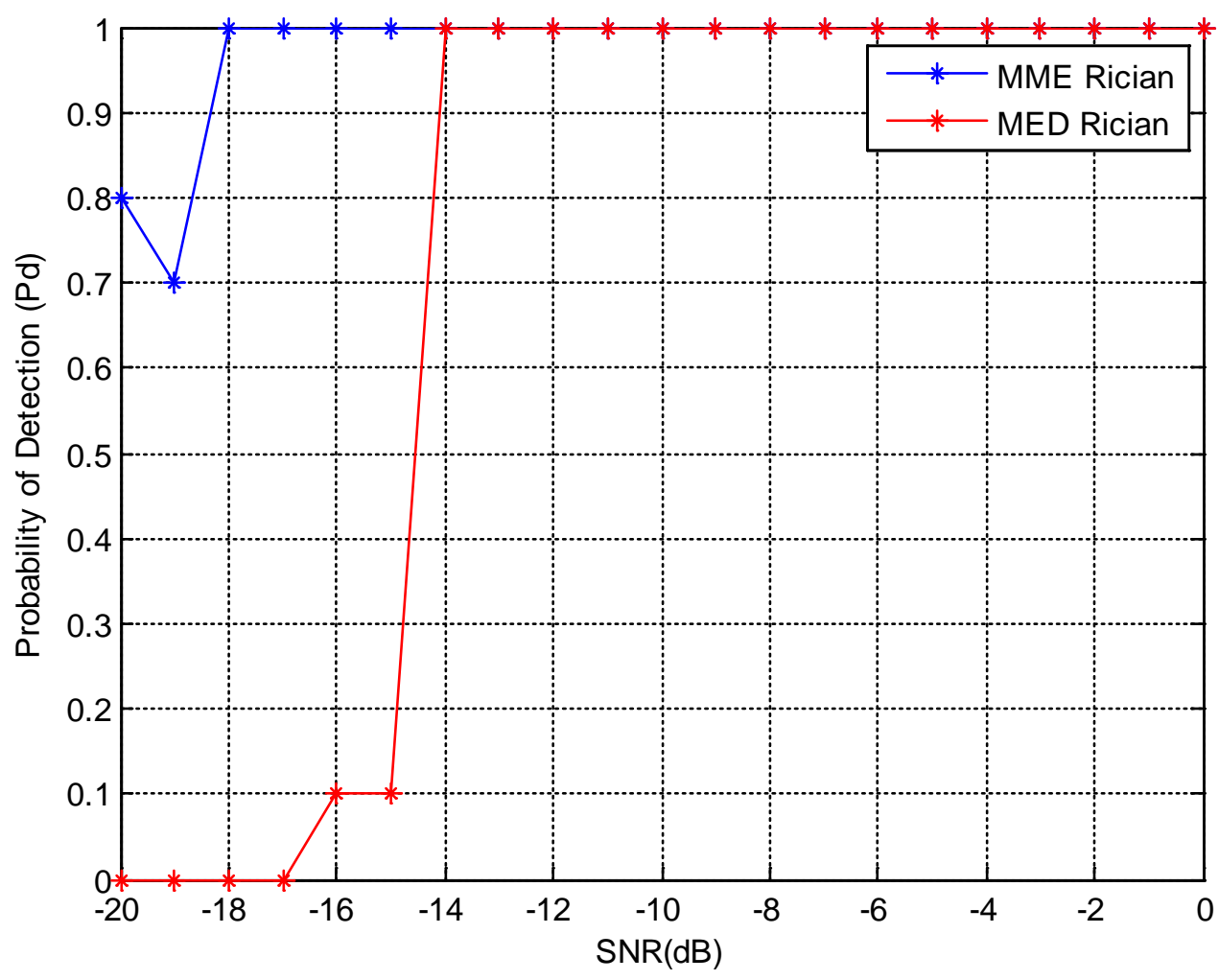

Figure 3. SNR vs. Probability of detection for MME and MED

Fig. 3 shows the probability of detection $P_{d}$ results for the MED and MME detection in Rician fading channel. The $P_{d}$ of MME is better than the $P_{d}$ of MED. Numerical evaluation of comparison of MME and MED is given in the Table 4.

Table 4. Numerical analysis of ROC curve

\begin{tabular}{|c|c|c|}
\hline \multirow{2}{*}{ SNR (dB) } & \multicolumn{2}{|c|}{ Probability of Detection when $P_{f a}=\mathbf{0 . 1}$} \\
\cline { 2 - 3 } & MME & MED \\
\hline \hline-20 & 0.8 & 0 \\
\hline-18 & 1 & 0.1 \\
\hline-16 & 1 & 1 \\
\hline-14 & 1 & 1 \\
\hline-12 & 1 & \multicolumn{2}{|c|}{1} \\
\hline
\end{tabular}




\section{Conclusion}

There are distinct eigenvalue based spectrum sensing algorithms based on eigenvalues of the covariance matrix of the received signal. Random matrix theory is used to set the thresholds and obtain the probability of detection. These algorithms give better performance than other spectrum sensing algorithms. Eigenvalue based detection is combined with other detection methods to maximize the probability of detection and to explore advantages of each individual detection method. Further, this dual stage detection method can be made self adaptive and fully blind with the help of noise power estimation feedback. The literature shows a major focus on the implementation aspects of spectrum sensing rather than sensing time. To decrease sensing time a multi-taper two stage spectrum sensing i.e. combination of energy and eigenvalue based detection can be used. Multi-taper improves sensing time and a dual stage method maximizes the probability of detection hence improving the overall performance. Furthermore, different eigenvalue based spectrum sensing algorithm performance can be evaluated for different fading channel conditions.

\section{References}

[1] FCC, "Facilitating opportunities for flexible, efficient, and reliable spectrum use employing cognitive radio technologies, notice of proposed rulemaking and order," FCC 03-322. Dec. 2003.

[2] J. Mitola, G. Q. Maguire, “Cognitive radios: making software radios more personal,” IEEE Personal Communications. Vol.6, no.4, Pp. 13-18, Aug. 1999.

http://dx.doi.org/10.1109/98.788210

[3] S. Haykin, "Cognitive radio: brain-empowered wireless communications," IEEE Journal on Selected Areas in Communications, Vol.23, no.2, Pp. 201-220, Feb. 2005. http://dx.doi.org/10.1109/JSAC.2004.839380

[4] Zhai Xuping, Pan Jianguo, "Energy-detection based spectrum sensing for cognitive radio,” Wireless, Mobile and Sensor Networks (CCWMSN07) IET Conference IEEE, ISSN 0537- 9989, Pp. 944-947, Dec. 2007.

http://dx.doi.org/10.1049/cp:20070306

[5] V. Prithiviraj, B. Sarankumar, A. Kalaiyarasan, P. Praveen Chandru, N. Nandakumar Singh, "Cyclostationary analysis method of spectrum sensing for Cognitive radio," Wireless Communication, Vehicular Technology, Information Theory and Aerospace Electronic Systems Technology (Wireless VITAE) IEEE, Pp. 1-5, March 2011. http://dx.doi.org/10.1109/WIRELESSVITAE.2011.5940821

[6] S. Shobana, R. Saravanan, R. Muthaiah, "Matched Filter Based Spectrum Sensing on Cognitive Radio for OFDM WLANs,” International Journal of Engineering and Technology (IJET) IEEE, Vol.5, no.1, ISSN 0975-4024, Mar 2013. Available at: http://www.enggjournals.com/ijet/docs/IJET13-05-01-077.pdf

[7] Y. Zeng, Y.C. Liang, A. T. Hoang and R. Zhang, "A review on spectrum sensing for cognitive radio: challenges and solutions," EURASIP Journal on Advances in Signal Processing Springer, Vol.10, ISSN 1687-6180, Jan. 2010.

[8] S. Dikmese, J. L. Wong, A. Gokceoglu, E. Guzzon, M. Valkama and M. Renfors, 


\section{Macrothink}

Network Protocols and Algorithms

ISSN 1943-3581

2016, Vol. 8, No. 2

"Reducing Computational Complexity of Eigenvalue based Spectrum Sensing for Cognitive Radio,” Proc. CROWNCOM 2013, Pp. 61-67, July 2013.

http://dx.doi.org/10.1109/CROWNCom.2013.6636795

[9] A. Ikram and A. Rashdi, "Complexity analysis of eigenvalue based spectrum sensing techniques in cognitive radio networks,” APCC, Pp. 290-294, Oct. 2012.

http://dx.doi.org/10.1109/APCC.2012.6388149

[10] Y. Zeng, Y. chang Liang, "Eigenvalue-based spectrum sensing algorithms for cognitive radio, Communications,” IEEE Transactions on, Vol. 57, no.6, Pp.1784-1793, June 2009.

[11] Andreas Bollig, Rudolf Mathar, "MMME and DME: Two New Eigenvalue-Based Detectors for Spectrum Sensing in Cognitive Radio," Global Conference on Signal and Information Processing (GlobalSIP) IEEE, Pp. 1210-1213, Dec. 2013.

http://dx.doi.org/10.1109/GlobalSIP.2013.6737125

[12] Shree Krishna Sharma, Symeon Chatzinotas, and Bjorn Ottersten, "Maximum Eigenvalue Detection For Spectrum Sensing Under Corelated Noise,” Acoustics, Speech and Signal Processing (ICASSP) IEEE International Conference, Pp. 7268-7272, May 2014.

http://dx.doi.org/10.1109/ICASSP.2014.6855011

[13] Syed Sajjad Ali, Chang Liu, Minglu Jin, "Minimum Eigenvalue Detection for Spectrum Sensing in Cognitive Radio,” International Journal of Electrical and Computer Engineering (IJECE), Vol.4, ISSN 2088-8708, August 2014.

[14] Naresh Gunichetty, S M Hiremath, S K Patra, “Two Stage spectrum Sensing for Cognitive Radio using CMME," IEEE International Conference on Communication and Signal Processing-ICCSP, Apr. 2015.

http://dx.doi.org/10.1109/ICCSP.2015.7322666

[15] Mohamed Hamid, Niclas Bjorsell,Slimane Ben Slimane, "Energy and Eigenvalue-Based Combined Fully-Blind Self-Adapted Spectrum Sensing Algorithm," Vehicular Technology IEEE Transactions, Vol.65, ISSN 0018-9545, Pp. 630-642, February 2015.

http://dx.doi.org/10.1109/TVT.2015.2401132

[16] Pedro Alvarezk, Nuno Pratask, Antonio Rodriguesk, Neeli Rashmi Prasad, Ramjee Prasad, "Energy Detection and Eigenvalue Based Detection: An Experimental Study Using GNU Radio,” IEEE Wireless Personal Multimedia Communications (WPMC) International Symposium, ISSN: 1347-6890, Pp. 1-5, June 2011.

\section{Copyright Disclaimer}

Copyright reserved by the author(s).

This article is an open-access article distributed under the terms and conditions of the Creative Commons Attribution license (http://creativecommons.org/licenses/by/3.0/). 